\title{
Research on the Internet of Vehicles Data Query Method Based on
}

\section{Distributed Data}

\author{
Huabo Xiao \\ College of Information Engineering, Jiangxi University of Technology, Jiangxi Nanchang
}

Keywords: Data Reduction; Distributed Database; Internet of Vehicles

\begin{abstract}
Internet of vehicles makes the traffic control become intelligent. In internet of vehicles data exchanging, an important service is to check the OBU-On Board Unit and how to improve the data exchange speed has always been an important research direction. This paper divides the distributed database into pseudo host and client-side, which carries out data reduction on the pseudo host. A dazzling array of data is exchanged via wired connection so as to improve the data exchange speed.
\end{abstract}

\section{Introduction}

Internet of vehicle is a typical application in the Internet of Things based on application custom service of SOA structure. Car information resource is national strategic resources and its application is of vital importance for national economic activities and social management service, which also plays a supporting role in constructing the internet of Things. In internet of vehicle system, how to manage the car data is a key problem which needs to be solved. Internet car data storage and complicated query system, as background data processing subsystems manage all of the car information resources, which provide support for the query service as well as data analysis of cloud platform applications. The main technique point is to effectively manage the large amounts of data and meanwhile provide simple reliable query interface for the diversity of application.

Years of practical experience in foreign countries proves that in order to solve the traffic problem we cannot only depend on road construction the more important point is to develop modern Intelligent Transportation System (ITS). In Stockholm, a new intelligent tolling system greatly reduces the traffic volume and carbon emissions; in London, the congestion management system successfully reduces the traffic volume to the level in middle of 1980s; in Singapore, traffic forecasting system can help people develop program and manage the traffic throughout the city so as to prevent serious traffic jams. However, the current intelligent traffic management system still has obvious application bottlenecks because there are demerits in terms of its instantaneity, accuracy and flexibility which cannot meet the requirement of traffic planning and traffic management. Internet of vehicles, as a typical application of the internet of things makes the spatiotemporal data possible which is able to realize more intelligent traffic management.

The data exchanging in internet of vehicles can store large amounts of data and distribute the storage which can guarantee the data safety. Therefore, the data redundancy is prominent. At the same time, the frequent data exchanging makes damages on the data integrity and completeness. So, in order to accurately and quickly realize data exchanging, we have to carry out initial attribute reduction on the desired information. 
Attribute reduction is a process to reduce the core attributes while keeping classification capacity which is the core problem of Rough theory. The Rough theory is put forward by Pawlak and the attribute reduction theory, calculation and application are fully developed, including reduction algorithm based on positive region, discernibility matrix, information theory, rough set approach and reduction algorithm combining other data mining machine learning algorithms etc. These reduction theories are effective to those databases with a small amount of data and little changes. Yet, there is a huge limitation for those databases with large amounts of data. In order to be adapted to those centralized data or big data, Liu Zongtian puts forward incremental reduction algorithm based on rough set; Wang Jue comes up with the idea of distributed reduction algorithm based on discernibility matrix; F.Fu puts forward the incremental reduction algorithm based on elements set; M. Kryszkiewicz etc put forward reduction algorithm based on multiple decision-making system. What's more, Jan. G. Bazan etc put forward the concept and approaches of dynamic reduction.

This paper designs a data exchanging method based on reduction theory in the context of analogue-digital environment of internet of vehicles. When the vehicle terminals give an order, it search and query in the database so as to carry out reduction data and send out a simple data result. The stimulated result reveals that the query speed remains to be improved.

\section{Approaches}

This paper mainly adopts reduction theory, and the following formulas are used:

Definition 1: If any $\mathrm{R}$ in $\mathrm{r} \in \mathrm{R}$ cannot be divided out, then the equivalent relation group $\mathrm{R}$ is independent or $\mathrm{R}$ is not related.

Definition 2: Suppose $R$ is a equivalent relation group, $r \in R$ and if $\operatorname{IND}(R)=\operatorname{IND}(R-\{r\})$ the $r$ can be divided out in $R$; if $P=R-\{r\}$ is independent, then $P$ is a reduction of $R$.

Definition 3:Those in $\mathrm{R}$ cannot be divided out are called the cores, remarked as Core $(\mathrm{R})$.

Definition 4: function $d x: A \rightarrow V$, suppose $d x(a)=a(x)$, among which $a \in A, X U, x \in U$. That is to say $d x$ is the previous decision rule of $T$ and if $a \in C A$, the $d x \mid C$ is part of the rule for decision and if $\mathrm{a} \in \mathrm{D} \in \mathrm{A}$, the $\mathrm{dx} \mid \mathrm{D}$ is the result.

Definition 5: if any $y \neq x, d x|C=d y| C \rightarrow d x|D=d y| D$, the $d x$ is consistent or it is inconsistent.

Definition 6: if any decisions are consistent the $\mathrm{T}=(\mathrm{U}, \mathrm{A}, \mathrm{C}, \mathrm{D})$ shall be consistent or it is inconsistent.

When the vehicles send out query information, the information is not directly sent to all of the databases but to the closest database so there is a Pseudo secondary relation. The closest database is Pseudo-principal and others are secondary. When they search information in the pseudo-principal database, it will send information to the secondary databases so as to carry out data analysis and reduction. Finally, the data is sent to the vehicle terminal because the query mainly happens among wired databases. Therefore, the speed is quick and the reliability is high, the approach can be concluded in the following figure: 


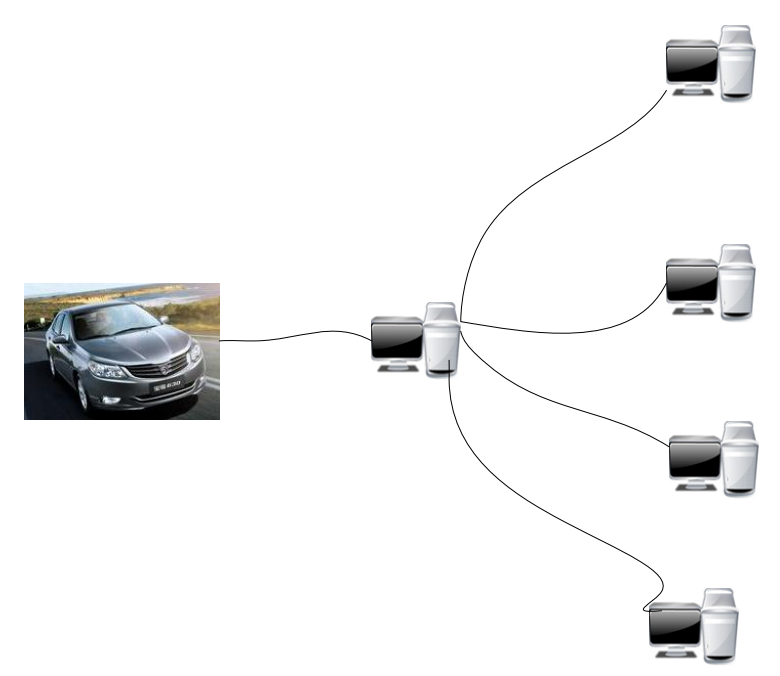

Fig. 1 Main framework

In internet of vehicles data analysis, the detailed process is shown in the following framework

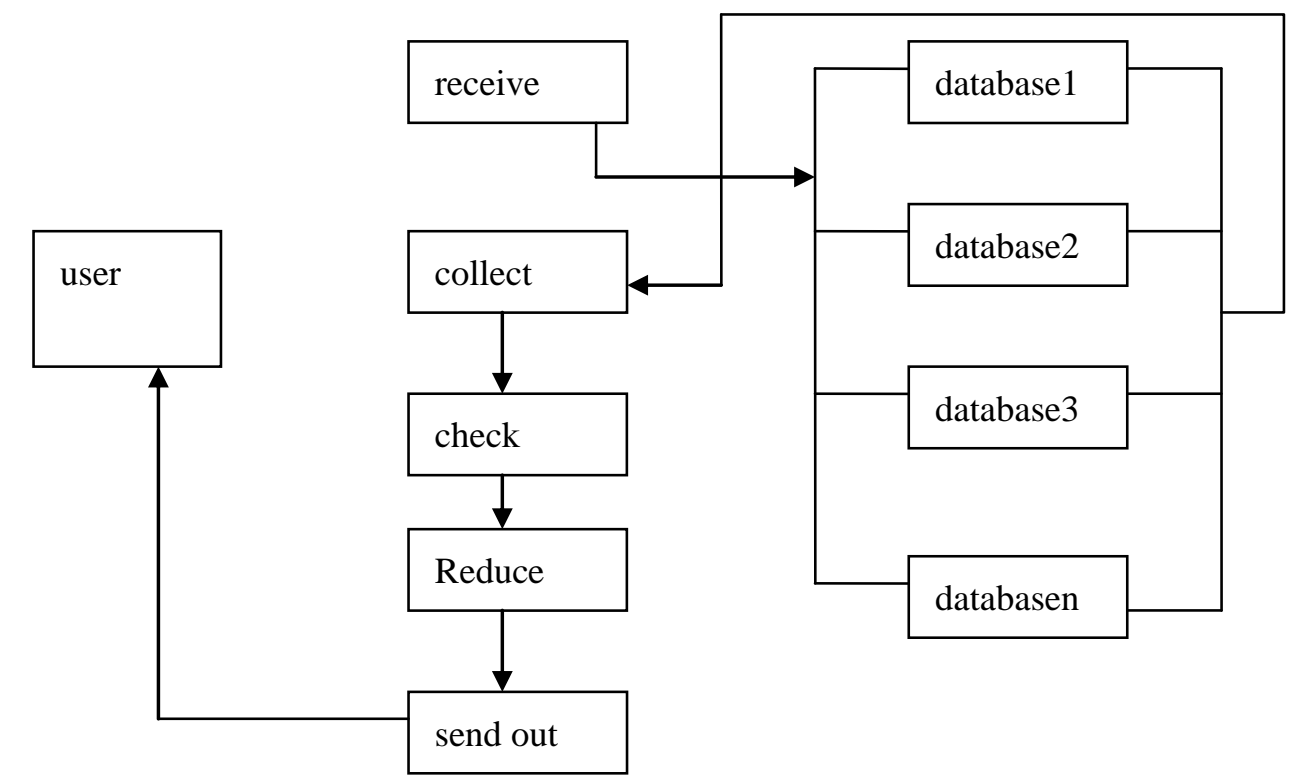

Fig. 2 Data flow

user is the client information needs;

receive: pseudo host receives the user information;

collect: pseudo host receives the required information sent by the distributed database

check: pseudo host carries out conflict detection

reduce: pseudo host carries out reduction calculation

send out: pseudo host send the user calculation result

Databasen: distributed database

\section{Conclusions}

There are two measurement indexes to query in the distributed database: the speed and accuracy. This paper is carried out from the perspective of speed. The data exchange happens during the period 
of wireless sending and receiving data as well as the period of pseudo host sending requirement, collecting results and carrying out data reduction.

Internet mainly depends on two communication technologies: short range wireless communication and mobile communication technology. The former is about RFID which is similar to WIFI while the latter is about GPRS, 3G, LTE and 4G etc.

In typical wireless networks the rate of transmission of $802.11 \mathrm{a}$ and $802.11 \mathrm{~g}$ is $11 \mathrm{Mbps}$ which is under ideal conditions. Under normal circumstances, especially the automotive condition, the average speed is $40 \%$ while the cable broadband can be calculated in arterial road which can be adopted to improve the exchanging speed.

Judging from the exchanging content, the difference of this approach lies in the point that this wireless exchanging content is reduced, which deletes the redundant and conflicted contents, greatly reducing the number of exchanging bytes. As a result, the exchanging speed is greatly improved.

\section{Acknowledgements}

This work was financially supported by project of Jiangxi University of Technology [No. XJXT1403] and Nature of Jiangxi University of Technology [No. ZR14YB03].The project of Technology Department of Jiangxi Province [No 20143BBM26048] also give us a lot of help.

\section{References}

[1] Z. Pawlak. Rough sets-Theoretical Aspect of Reasoning about Data. Dordrecht: Kluwer Academic Publishers, 1991.

2. Liu Qing. Rough sets and rough reasoning. Beijing: Science Press, 2001.

3. Wang Guoyin. Rough theory and knowledge acquisition. Xian: Xian Jiaotong University Press, 2001.

4. Zhang Wenxiu. Mi Jusheng, Wu Weijie. Knowledge Reductions in Inconsistent Information Systems. Chinese Journal of Computers, 26(1):12-18, 2003.

5. Miao Duoqian, Wang Guoyin, Liu Qing. Granular Computing: Past, Present, Future and Prospect. Science Press, 2007.8

[6] K. Thangavel, A. Pethalakshmi. Dimensionality reduction based on rough set theory: A review. Applied Soft Computing. 9:1-12, 2009.

[7] Mu Zhendong, Zou Weigang, Jiang Shunliang. Discussion on data reduction. Computer and Modernization. 5th series of 2003.

[8]X.Hu, N.Cercone. Learning in relational databases: a rough set approach. Computational Intelligence, 11(2):323-337, 1995.

9. Ye Dongyi, Chen Zhaojiong. A new discernibility matrix and its computation of a core, Chinese Journal of Electronics, 30(7):1086-1088, 2002.

[10]D.Ye, Z.Chen. Inconsistency classication and discernibility-matrix-based approaches for computing an attribute core. In Proceedings of the 9th International Conference on Rough Sets, Fuzzy Sets, Data Mining, and Granular Computing (RSRDGrC2003), LNAI2639, 269-273. China, 2003. 